\title{
Dynamics of small unilamellar vesicles
}

Cite as: J. Chem. Phys. 148, 104901 (2018); https://doi.org/10.1063/1.5009424

Submitted: 16 October 2017 . Accepted: 23 February 2018 . Published Online: 12 March 2018

Ingo Hoffmann, Claudia Hoffmann, Bela Farago, Sylvain Prévost (D), and Michael Gradzielski
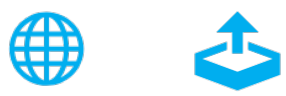

View Online

Export Citation

\section{ARTICLES YOU MAY BE INTERESTED IN}

Particle-based membrane model for mesoscopic simulation of cellular dynamics The Journal of Chemical Physics 148, 044901 (2018); https://doi.org/10.1063/1.5009107

Structure and dynamics of polyelectrolyte surfactant mixtures under conditions of surfactant excess

The Journal of Chemical Physics 145, 124901 (2016); https://doi.org/10.1063/1.4962581

Effect of interlamellar interactions on shear induced multilamellar vesicle formation The Journal of Chemical Physics 147, 034905 (2017); https://doi.org/10.1063/1.4994563
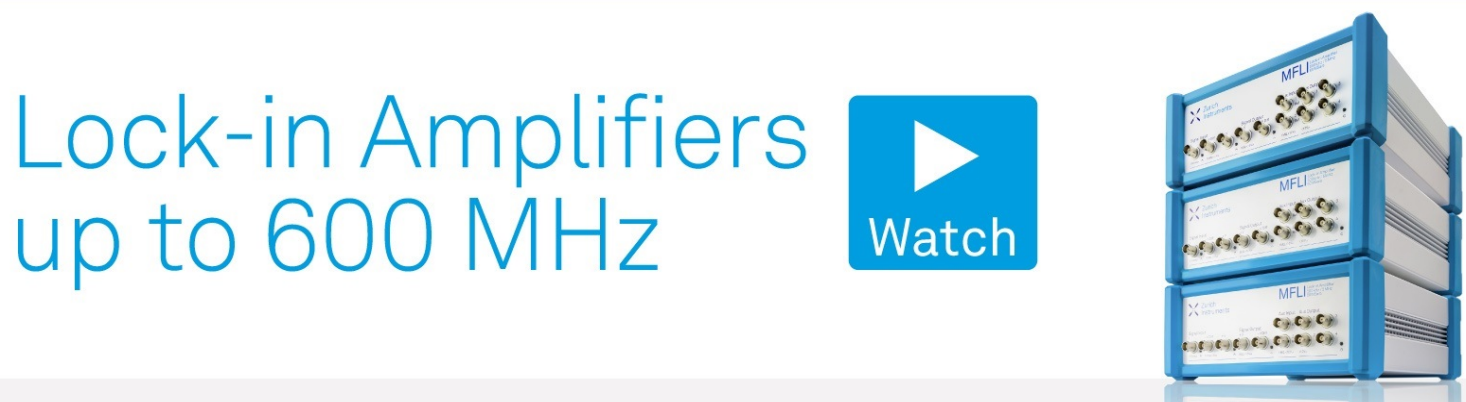


\title{
Dynamics of small unilamellar vesicles
}

\author{
Ingo Hoffmann, ${ }^{1,2, a)}$ Claudia Hoffmann, ${ }^{1}$ Bela Farago, ${ }^{2}$ Sylvain Prévost, ${ }^{1,2,3}$ \\ and Michael Gradzielski ${ }^{1, b)}$ \\ ${ }^{1}$ Stranski-Laboratorium für Physikalische und Theoretische Chemie, Institut für Chemie, Technische Universität \\ Berlin, Straße des 17. Juni 124, Sekr. TC 7, D-10623 Berlin, Germany \\ ${ }^{2}$ Institut Max von Laue-Paul Langevin (ILL), 71 Avenue des Martyrs, CS 20156, F-38042 Grenoble Cedex 9 , \\ France \\ ${ }^{3}$ Helmholtz-Zentrum Berlin, Hahn-Meitner-Platz 1, D-14109 Berlin, Germany
}

(Received 16 October 2017; accepted 23 February 2018; published online 12 March 2018)

In this paper, we investigate the dynamics of small unilamellar vesicles with the aid of neutron spinecho spectroscopy. The purpose of this investigation is twofold. On the one hand, we investigate the influence of solubilised cosurfactant on the dynamics of the vesicle's surfactant bilayer. On the other hand, the small unilamellar vesicles used here have a size between larger vesicles, with dynamics being well described by the Zilman-Granek model and smaller microemulsion droplets which can be described by the Milner-Safran model. Therefore, we want to elucidate the question, which model is more suitable for the description of the membrane dynamics of small vesicles, where the finite curvature of the bilayer is felt by the contained amphiphilic molecules. This question is of substantial relevance for our understanding of membranes and how their dynamics is affected by curvature, a problem that is also of key importance in a number of biological questions. Our results indicate the even down to vesicle radii of $20 \mathrm{~nm}$ the Zilman-Granek model appears to be the more suitable one. Published by AIP Publishing. https://doi.org/10.1063/1.5009424

\section{INTRODUCTION}

Closed surfactant bilayers are called vesicles. They have attracted quite some interest over the past decades, and as opposed to many other carrier systems, they can be used for the delivery of both hydrophilic and hydrophobic molecules. ${ }^{1-9}$ There is also quite some interest in them, as they can be used as simple model systems for cells, especially when they consist of lipids instead of synthetic surfactants, when such vesicles are also called liposomes. ${ }^{2,4,7,10-12}$

As a significant part of their stability stems from the undulation motion of their membrane, ${ }^{13}$ there is quite some relevance to the study of their membrane dynamics and its change when loading the membrane with another molecule. In general, the membrane elasticity is a key property of such bilayer systems and is quantitatively described by its bending moduli, the mean bending modulus $\kappa$ and the Gaussian modulus $\bar{K} .{ }^{14}$ Accordingly, there have been many studies to determine the bending moduli of membranes often via optical methods ${ }^{15,16}$ or light scattering, ${ }^{17}$ and these approaches have been reviewed comprehensively, ${ }^{18-20}$ showing that in general the bending rigidity depends strongly on the type of membrane studied (and for instance a stiffening is observed upon the introduction of cholesterol into phospholipid bilayers ${ }^{21}$ ). An interesting but less easily accessible method to study membrane dynamics is neutron spin-echo (NSE) spectroscopy ${ }^{22,23}$ that allows us to follow membrane fluctuations in the range of $1-20 \mathrm{~nm} \cdot{ }^{24,25}$ NSE is complementary to dynamic light

\footnotetext{
a)Electronic mail: hoffmann@ill.fr

b)Electronic mail: michael.gradzielski@tu-berlin.de
}

scattering that probes length scales of several hundreds of $\mathrm{nm}$. This is relevant as the bending properties of membranes and layers are known to depend on the length scale probed, ${ }^{26}$ becoming smaller with smaller probed length scale. NSE has the advantage of probing rather directly the mean bending modulus that can be derived from the intermediate scattering function $\mathrm{S}(\mathrm{Q}, \mathrm{t})$.

A particularly interesting parameter for the understanding of the bilayer elasticity is its thickness, and there are some studies investigating its influence ${ }^{27-31}$ on the membrane rigidity. A study on bilayer vesicles assembled from amphiphilic diblock copolymers showed that the mean bending rigidity $\kappa$ for large polymersomes scales with the hydrophobic membrane thickness to the power two. ${ }^{32}$ Most other work has been dealing with surfactant bilayers which are loaded with rather large amounts of other compounds, and therefore, the two monolayers are decoupled and a decrease of the bending rigidity is observed. However, initially an increase is observed, ${ }^{27}$ as the bilayer behaves as a thicker membrane. Here, we want to focus on that region and investigate the scaling of the bending rigidity $\kappa$ with the bilayer thickness when relatively small amounts of other molecules are added. If they behave as an isotropic material, they should scale as $\kappa \propto d^{3},{ }^{33}$ while as long as they behave as a true bilayer $\kappa$ should scale as $\kappa \propto d^{2} .{ }^{14,34}$ Szleifer et al. ${ }^{35}$ on the other hand, predicted a strong reduction by more than a factor 2 upon addition of relatively small amounts of shorter chains to a bilayer based on their conformational entropy with only relatively little impact on the membrane thickness.

Recently Mell et al. ${ }^{36}$ investigated whether the ZilmanGranek model or the Milner-Safran model is more appropriate to describe the dynamics of relatively large liposomes and 
found better agreement with the Zilman-Granek model. However, while the Zilman-Granek model has been largely successful in describing the dynamics in relatively large membrane structures with sizes of $50 \mathrm{~nm}$ and more, small microemulsion droplets with sizes on the order of $5 \mathrm{~nm}$ are well described by the Milner-Safran model. ${ }^{37,38}$ It is reasonable to assume that there is a size below which the Milner-Safran model works better, as apparently it applies well to microemulsion droplets. In order to elucidate this interesting point at which size vesicles become small enough to be better treated with the MilnerSafran model, we investigated the dynamics of small unilamellar vesicles, which are larger than microemulsion droplets but smaller than the vesicles usually studied before.

For this purpose, we chose a well-studied system based on sodium oleate or isostearate, where by addition of medium chain alcohols such as octanol ${ }^{39,40}$ or decanol, ${ }^{41}$ spontaneous formation of rather monodisperse unilamellar vesicles has been observed. These systems contain an excess of alcohol compared with the surfactant (typically a molar ratio of 2-3:1 of alcohol to oleate) and form at higher concentration a well-ordered vesicle gel. However, upon dilution with water, one ends up in an isotropic solution that contains well-defined unilamellar vesicles. Such vesicles were then chosen for our NSE experiments to determine the mean bending modulus and to elaborate whether the Milner-Safran model or the Zilman-Granek model is more suited to describe them.

\section{MATERIALS AND METHODS}

\section{A. Materials}

Oleic acid (purum 98\%, Fluka) and isostearic acid (Emersol 874) were dissolved in $\mathrm{D}_{2} \mathrm{O}$ (99.9\% Euriso-top) with equimolar amounts of sodium hydroxide ( $98 \%$ puris, Fluka) to yield $80 \mathrm{mM}$ stock solutions of sodium oleate and sodium isostearate. The surfactant stock solutions were added to the appropriate amounts of octanol (per synthesis, Merck) and vigorously stirred overnight to obtain solutions of small vesicles.

\section{B. Methods}

Small angle neutron scattering ${ }^{42}$ (SANS) measurements were performed on the instrument $\mathrm{V}^{43,44}$ at HelmholtzZentrum Berlin (HZB) using sample to detector distances of 1,4 , and $15.85 \mathrm{~m}$ and a wavelength $\lambda=0.457 \mathrm{~nm}$ allowing to cover a $Q$ range from 0.036 to $5.71 / \mathrm{nm}$, where $Q$ is the magnitude of the scattering vector $Q=4 \pi / \lambda \sin (\theta / 2)$ and $\theta$ is the scattering angle. The software package BerSANS ${ }^{45}$ was used for data reduction.

The SANS curves were modeled as slightly polydisperse ( $10 \%$ relative standard deviation in radius) hollow spheres with a single shell. The form factor for such a spherical shell reads

$$
P\left(Q, R_{i}, \Delta S L D_{i}\right)=\left(\sum_{i=0}^{1} F\left(Q, R_{i}, \Delta S L D_{i}\right)\right)^{2},
$$

where $R_{0}$ is the radius of the aqueous core, $R_{1}$ is the outer radius, and the thickness of the surfactant bilayer is given by
$d_{s}=R_{1}-R_{0}$ and the $\triangle S L D_{i}$ are the differences in scattering length density (SLD) going from $R>R_{i}$ to $R<R_{i}$. The amplitude $F$ is given by

$$
F\left(Q, R_{i}, \Delta S L D_{i}\right)=\frac{4 \pi R_{i}^{3}}{3} \Delta S L D_{i} 3 \frac{\sin \left(Q R_{i}\right)-Q R_{i} \cos Q R_{i}}{\left(Q R_{i}\right)^{3}}
$$

and the scattering intensity for a non-interacting, monodisperse system is given by $I={ }^{1} N P\left(Q, R_{i}, \Delta S L D_{i}\right)+I_{\text {inc }}$, where ${ }^{1} N$ is the particle number density and $I_{i n c}$ is the incoherent scattering contribution which has been subtracted. In a monodisperse system, ${ }^{1} N$ is given by ${ }^{1} N=\phi / V(R)$, with the volume fraction $\phi$ and the volume of the particles $V(R)$. In a polydisperse system, it is given by

$$
{ }^{1} N=\frac{\phi}{\int_{0}^{\infty} f(R) V(R) \mathrm{d} R},
$$

where $f(R)$ is the size distribution of the vesicles. Here, we used the log-normal size distribution

$$
f(x, \mu, \sigma)=\frac{1}{\sqrt{2 \pi} \sigma \cdot x} \exp \left(-\frac{(\ln (x / \mu))^{2}}{2 \sigma^{2}}\right),
$$

with arithmetic mean $M=\mu \exp \left(\sigma^{2} / 2\right)$ and its standard deviation is given by $S D=\mu \sqrt{\left(\exp \left(\sigma^{2}\right)-1\right)} \exp \left(\sigma^{2} / 2\right)$.

For a system with interactions, it is necessary to introduce a structure factor $S(Q)$, and in the local monodisperse approach, ${ }^{46}$ the intensity for a polydisperse, interacting system now reads

$I={ }^{1} N \int_{0}^{\infty} f(R, \mu, \sigma) P\left(Q, R_{i}, \Delta S L D_{i}\right) S(Q, R, \phi) \mathrm{d} R+I_{\text {inc }}$,

where the hard-sphere structure factor in the Percus-Yevick approximation $^{47}$ has been used. See the supplementary material for details.

Neutron spin-echo ${ }^{22,23}$ (NSE) measurements were performed on the instrument IN $15^{48}$ at Institut Laue-Langevin (ILL, Grenoble, France). Using wavelengths of 10 and $17 \AA$, maximum Fourier times of 50 and 200 ns were reached covering a $Q$ range from 0.13 to $1.31 / \mathrm{nm}$.

NSE accesses the intermediate scattering function $S(q, t)$. To get a general idea about the system's behaviour, data can be described with an apparent diffusion coefficient $D_{a p p}$,

$$
S(Q, t)=\exp \left(-D_{a p p} Q^{2 t}\right)
$$

\section{THEORY}

The general form of $S(Q, t)$ for the description of membrane dynamics in diffusing systems is given by

$$
S(Q, t)=\exp \left(-D(Q) Q^{2} t\right)\left((1-A(Q))+A(Q) S_{\text {und }}(Q, t)\right),
$$

where $D(Q)$ is the $Q$-dependent translational diffusion coefficient

$$
D(Q)=D_{0} / S(Q),
$$

with the translational diffusion coefficient at infinite dilution $D_{0}$, which is related to the size of the object via the StokesEinstein equation

$$
D_{0}=\frac{k_{B} T}{6 \pi \eta R_{H}}
$$


with the hydrodynamic radius $R_{H}$, solvent viscosity $\eta$, Boltzmann constant $k_{B}$, and temperature $T$. $S_{\text {und }}(Q, t)$ describes the undulation motion of the membrane.

For the description of the membrane dynamics in microemulsion droplets, the Milner-Safran model ${ }^{37,38,49-52}$ has proven successful. In the framework of this model membrane motions are described as spherical harmonics and $S_{\text {und }}$ is given by a series of simple exponentials $S_{\text {und,MS }}$ $=\sum_{l=2}^{\infty} a_{l} \exp \left(-\Gamma_{M S, l} t\right)$ with relaxation rates $\Gamma_{M S, l}$

$$
\Gamma_{M S, l}=\frac{\kappa}{\eta R^{3}} \frac{l(l+1)-6+4 w-3 \frac{\bar{\kappa}}{\kappa}-\frac{3 k_{B} T}{4 \pi \kappa} \Phi(\phi)}{Z(l)},
$$

where $\kappa$ is the bending rigidity, $\bar{\kappa}$ is the saddle splay modulus, $\phi$ is defined as is accounting for the entropy of mixing and can be approximated as $\Phi(\phi)=\frac{1}{\phi}(\phi \ln (\phi)+(1-\phi) \ln (1-\phi)), \phi$ is the volume fraction of the dispersed microemulsion droplets, $Z$ is given by $Z(l)=\frac{(2 l+1)\left(2 l^{2}+2 l-1\right)}{l(l+1)(l+2)(l-1)}$, and $w=c_{0} R$, where $c_{0}$ is the spontaneous curvature. The sum in Eq. (10) starts at 2 as the zero order term corresponds to size fluctuations, which are too slow for NSE to capture and the first order term corresponds to diffusion. The non-normalised amplitudes are given by

$$
a_{l}=\frac{k_{B} T}{\kappa\left((l+2)(l-1)\left(l(l+1)-6+4 w-3 \frac{\bar{K}}{\kappa}-\frac{3 k_{B} T \Phi(\phi)}{4 \pi \kappa}\right)\right)} .
$$

The amplitude of the dynamic contribution $A(Q)$ is predicted to show a maximum around the form factor minimum, and in fitting experimental data using the model usually only a single exponential term is kept to account for membrane dynamics, ${ }^{52}$ as both the amplitudes quickly decrease with $l$ (see Fig. S1 of the supplementary material) and the $\Gamma_{M S, l}$ quickly increase and therefore move out of the dynamic range. Applying the model to the membrane dynamics of larger objects such as large unilamellar vesicles, it is usually assumed that the relaxation at a given $Q$ are dominated by undulations of the corresponding length scale resulting in a single exponential accounting for the membrane dynamics with a relaxation rate $\Gamma=\frac{\kappa}{4 \eta} Q^{3} \cdot{ }^{36,53,54}$ See the electronic supplementary material [Eqs. (S3)-(S5)] for more details.

The Zilman-Granek model ${ }^{55,56}$ on the other hand predicts a stretched exponential decay of $S_{\text {und }}(Q, t)$ with a stretch exponent of $2 / 3$,

$$
S_{\text {und }, Z G}(Q, t)=\exp \left(-\left(\Gamma_{Z G} Q^{3} t\right)^{2 / 3}\right),
$$

with $\Gamma_{Z G}=0.025 \gamma \sqrt{\frac{k_{B} T}{\kappa}} \frac{k_{B} T}{\eta}$ and for $\kappa / k_{B} T \gg 1, \gamma \approx 1$. As it was developed for large bilayer structures where translational diffusion is negligible, no explicit prediction is made for $A(Q)$.

The concept of both models is similar in that they both start from a Helfrich bending Hamiltonian ${ }^{14}$ but the MilnerSafran model only takes into account the smallest wave vector undulation, which corresponds to the radius of the particle [first fraction in Eq. (10)]. The Zilman-Granek model on the other hand results from an integration over all undulation wave vectors between the length scale of the particle and a lower cut-off molecular length scale. Therefore, the Milner-Safran model is suited for the description of relatively small droplets, while the Zilman-Granek model is suited for the description of larger membrane structures.
The most prominent difference between the 2 models is the scaling of $\Gamma$ with $\kappa$. While the Milner-Safran model pre$\operatorname{dicts} \Gamma_{M S} \propto \kappa$, the Zilman-Granek model predicts $\Gamma_{Z G} \propto 1 / \sqrt{\kappa}$, and it was its benefit that it managed to give an explanation for the previously observed scaling of the relaxation rate with $\kappa \cdot{ }^{17,24}$ The second difference is the pronounced increase of the amplitude $A$ around the form factor minimum in the Milner-Safran model. While no explicit prediction is made by the Zilman-Granek model, as it is the result of averaging over a range of wave vectors, a more smooth behaviour is expected and also predicted by the Milner-Safran model at higher $Q$ and when taking into account larger undulation wave vectors. $^{36}$

The third difference is the shape of the function. However, differentiating between a stretched exponential with a stretch exponent of $2 / 3$ and a simple exponential is not necessarily straightforward especially, when combined with another exponential as in Eq. (7).

While the scaling of $\Gamma$ with $\kappa$ is correctly predicted, the absolute values of $\kappa$ obtained from NSE measurements have often shown to be wrong and a number of approaches exist to correct them. ${ }^{25,57-63}$ For example, Seifert and Langer ${ }^{60}$ took into account contributions from lateral flow in the membrane and interbilayer friction. ${ }^{64}$ Watson and Brown ${ }^{62}$ adapted that theory to the framework of the Zilman-Granek theory, and the measured, effective bending rigidity is given by $\kappa_{e f f}=\kappa+2 d^{2} k$, where $k$ is the monolayer compressibility modulus and $d$ is the height of the monolayer neutral surface. Monkenbusch et al. ${ }^{61}$ explicitly evaluated the integrals which arise in the derivation of Eq. (12) to account for the finite size of the film and the molecules which comprise it. As here, we are mainly interested in relative changes in $\kappa$, we will limit ourselves to the simplest correction, which consists of using an effective solvent viscosity $\eta_{\text {eff }}=3 \eta,{ }^{57-59}$ which previously led to realistic values of $\kappa$.

\section{RESULTS AND DISCUSSION}

For our experiments, we choose well-defined, spontaneously forming vesicles that are not too concentrated, so the individual vesicles are able to move and undulate freely. This was accomplished by using a surfactant $(\mathrm{Na}$ oleate or $\mathrm{Na}$ isostearate) concentration of $80 \mathrm{mM}$, which is well below the gelation concentration of $\sim 100 \mathrm{mM}$ but not too dilute as then the vesicles stop being well-defined. ${ }^{1}$ The octanol concentration was varied in the range of $150-300 \mathrm{mM}$, where formation of unilamellar vesicles should occur, in order to vary systematically the thickness and composition of the bilayer. We performed SANS measurements to verify the presence of vesicles in the samples and precisely determine their structure, especially the thickness of the surfactant layer as a function of surfactant to cosurfactant ratio. The SANS curves taken for samples with $80 \mathrm{mM}$ Na oleate and 150, 225, and $300 \mathrm{mM}$ 1-octanol (corresponding to cosurfactant mass fractions of 2,3 , and 4 wt. $\%$ or surfactant to cosurfactant volume ratios $\phi_{s} / \phi_{\text {Octanol }}$ of $1.04,0.68$, and 0.52 ) and $80 \mathrm{mM} \mathrm{Na}$ isostearate and $225 \mathrm{mM} 1$-octanol (corresponding to a cosurfactant mass fraction of $3 \mathrm{wt}$. \% or a surfactant to cosurfactant volume ratio 


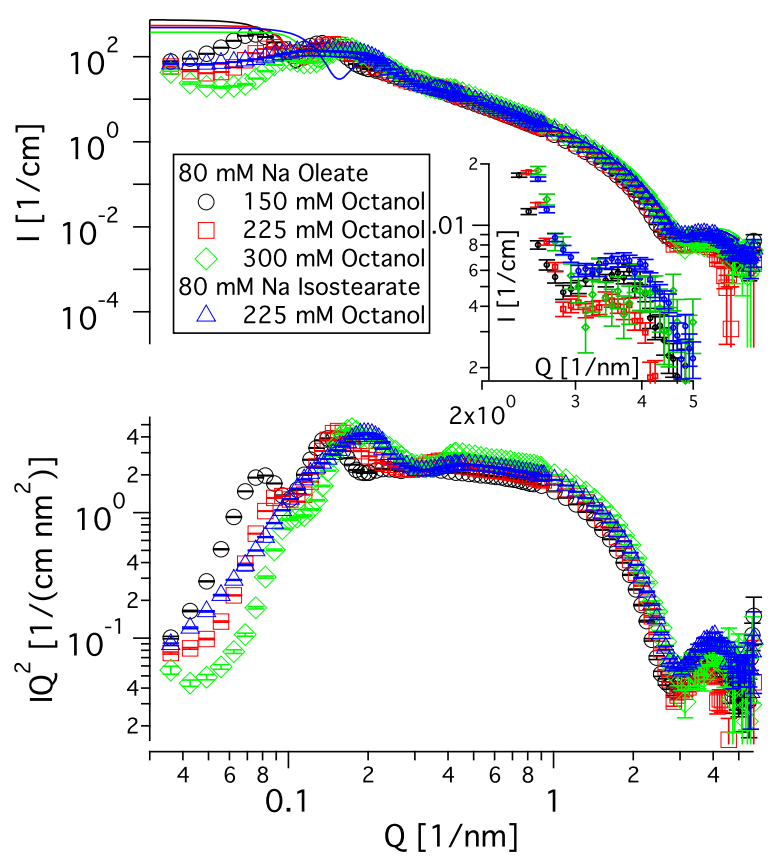

FIG. 1. Top: SANS curves of samples indicated in the graph with fits according to Eq. (5); bottom: Kratky plot $\left(I Q^{2}\right.$ vs. $\left.Q\right)$ of the same SANS curves. Inset: Magnification of the region between 2 and $51 / \mathrm{nm}$ showing the form factor minimum due to the membrane thickness. The slight shift in its position shows that the membrane thickness changes slightly.

$\phi_{s} / \phi_{\text {Octanol }}$ of 0.66) are given in Fig. 1 and confirm the presence of small vesicles with radii between 20 and $40 \mathrm{~nm}$ (seen by the form factor minimum around $0.11 / \mathrm{nm}$ ), which can be seen from the $Q^{-2}$ slope at intermediate $Q$ and the resulting plateau in the Kratky plot (see Fig. 1, bottom). The minimum at high $Q$ (around $31 / \mathrm{nm}$ ) is slightly different between the various samples (also best seen in the Kratky plot), which means that the membrane thickness $\left(d_{\text {shell }}=2 \pi / Q_{\text {min }}\right)$ varies with different ratios between the surfactant and octanol. In Fig. 2, it can be seen that the layer thickness determined from the position of the high- $Q$ minimum decreases linearly with the addition of the cosurfactant octanol, which has a significantly shorter chain than the surfactant itself. This means

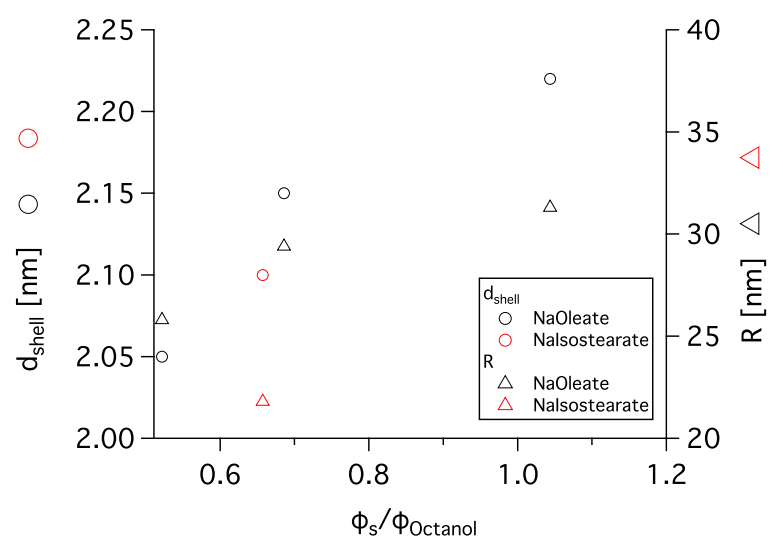

FIG. 2. Thickness of the surfactant layer (left axis, circles) and vesicle radius (right axis, triangles) as a function of surfactant to cosurfactant ratio. The surfactant layer thickness $d_{s}$ increases linearly with the surfactant to cosurfactant volume ratio. The overall size of the vesicles increases as well. that the concentration of octanol is a suitable tool for controlling the thickness of the vesicle bilayers. The values for the measurement with sodium oleate and sodium isostearate seem to fall on the same line for $d_{\text {shell }}$, but both systems differ with respect to the radius. The smaller radius for sodium isostearate indicates that it has a more flexible membrane that tends to a smaller spontaneous curvature. The double peak at low $Q$ (see Fig. 1) has been observed previously for similar systems ${ }^{1}$ and is due to the overlap between form factor minimum and structure factor maximum. While the combination of hard sphere structure factor and a hollow sphere manages to qualitatively reproduce the double peak with a dip in the middle at low $Q$, it fails to quantitatively describe the lowest $Q$ part; however, this is beyond the scope of this paper and of no relevance, as the NSE measurements do not reach such small $Q$ values. Anyway this $Q$-range probes already largely the interactions between the vesicles and therefore would be less relevant for determining the shape fluctuations of the vesicles.

After having elucidated the structure of the vesicles, we proceeded to perform NSE measurements in the $Q$-range of $0.13-1.31 / \mathrm{nm}$ to investigate their dynamics. The measured NSE curves (see Fig. 3 and Figs. S2-S4 of the supplementary material) show non-exponential relaxation behaviour, as can be seen from their curvature in the log-lin plot.

A preliminary analysis using Eq. (6) yields relatively constant values of $D_{a p p}$ (see Fig. 4). Additionally there is a constant offset between the values obtained from 10 or $17 \AA$ measurements which indicates a constant non-exponentiality over the whole $Q$ range. Only at the lowest $Q, D_{a p p}$ increases. At these values, however $S(Q, t)$ only decays from 1 to more than 0.9 , and this behaviour should not be mistaken to be due to the increase in $D_{\text {app }}$ around the form factor minimum predicted by the Milner-Safran model, especially as it is similarly observed for the sample with sodium isostearate which forms smaller vesicles and therefore has its form factor minimum at somewhat higher $Q$. A more likely source of this effect is de Gennes narrowing ${ }^{65}$ [see Eq. (8)]. The absolute values obtained are not in agreement with the values expected for simple translational diffusion of vesicles of the sizes found in SANS. For

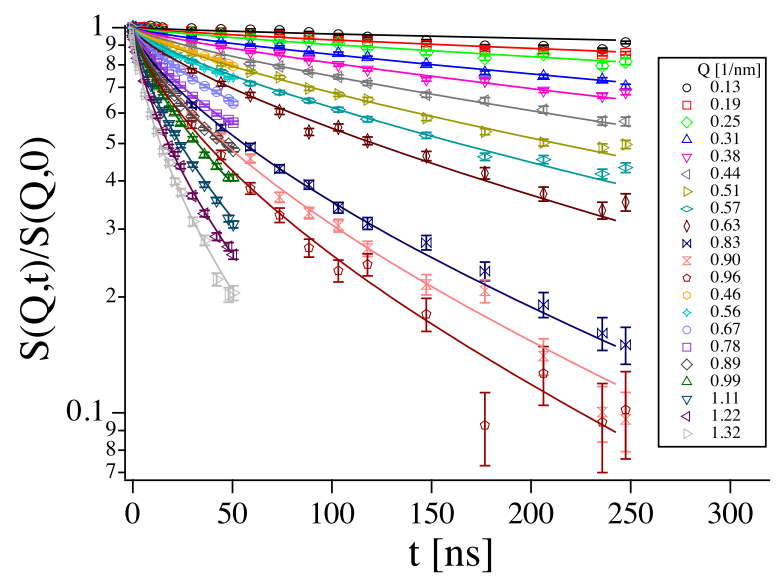

FIG. 3. $S(Q, t)$ of $80 \mathrm{mM}$ sodium oleate with $3 \mathrm{wt} \% 225 \mathrm{mM}$ octanol; fits using the Zilman-Granek model: Eq. (7) with Eqs. (8) and (12); fits are in good agreement with the data. It can be seen that $S(Q, t)$ is nonexponential throughout the whole $Q$-range. 


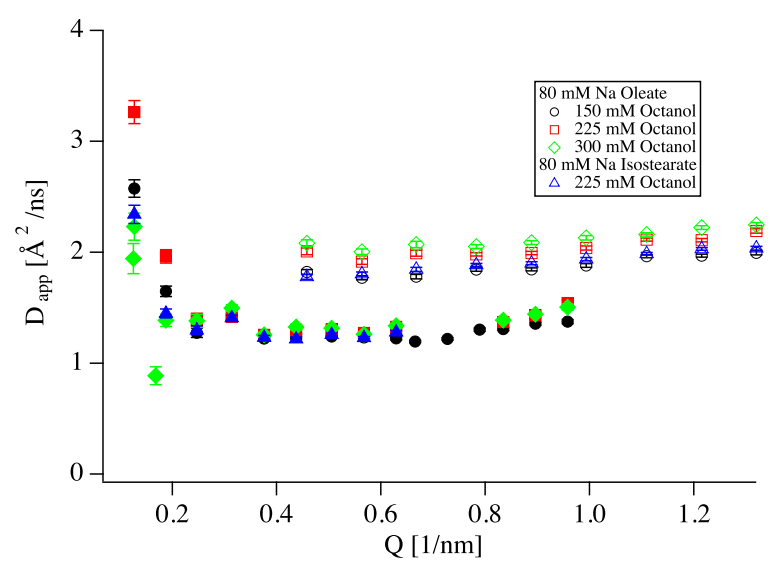

FIG. 4. $D_{a p p}$ obtained from fitting Eq. (6) to NSE data indicated in the graph. The values are relatively constant over the whole $Q$ range, and there is a constant offset between 10 (higher values, open symbols) and $17 \AA$ wavelength data (lower values, filled symbols), indicating non-exponentiality.

example, for vesicles with radii between 20 and $40 \mathrm{~nm}$ Eq. (9) would give diffusion coefficients between 1 and $0.5 \AA^{2} / \mathrm{ns}$, which are substantially lower than the values obtained, and obviously the membrane dynamics give a significant contribution to $S(Q, t)$ at all investigated $Q$ values. The fact that there is no pronounced change with $Q$ and that $S(Q, t)$ has a similarly non-exponential shape over the whole $Q$-range indicates that the vesicles are already large enough for the Zilman-Granek model to be the more appropriate description. Specifically, the lack of an increase of $D_{a p p}$ toward smaller $Q$ shows that using the Milner-Safran model simply using a relaxation rate $\Gamma=\frac{K}{4 \eta} Q^{3}$ is inappropriate, here.

Therefore, the NSE data were fitted using Eq. (7) with Eq. (12), and the contribution for translational diffusion was described by Eq. (8) with Eq. (S1) using the radius obtained from SANS. The fits are in good agreement with the data (see Fig. 3), and it can also be seen that $S(Q, t)$ is nonexponential at all $Q$.

Nevertheless, the data were also fitted with the MilnerSafran model using the same translational contribution but replacing the stretched exponential of Eq. (12) with a simple exponential with a relaxation rate which does not depend on $Q$ as predicted by Eq. (10), and significantly worse fit results are obtained (see Fig. 5) with a $\chi^{2}$ higher by roughly a factor 5 (see Fig. 6). Not neglecting higher order terms up to $l=5$ in the Milner-Safran model only slightly improves the situation, and including even higher order terms have no significant effect as their amplitude is extremely small. See Sec. II and Fig. S1 of the supplementary material for details. The inset in Fig. 6 shows that the Zilman-Granek model also yields a well-defined minimum for the relaxation rate $\Gamma_{Z G}$. In addition, the amplitude of the undulation contribution was left as a free parameter and while it shows a slight increase with $Q$ for the Zilman-Granek model, a strong increase is observed for the Milner-Safran model, and it fails to produce the predicted maximum around the form factor minimum (see Fig. S5 of the supplementary material). Furthermore the values for $\kappa$ [ignoring the corrections in the second fraction of Eq. (10)] obtained with the Milner-Safran model do not show a clear trend in $d_{\text {shell }}$ (see Fig. S6 of the supplementary material), which would not really be expected.

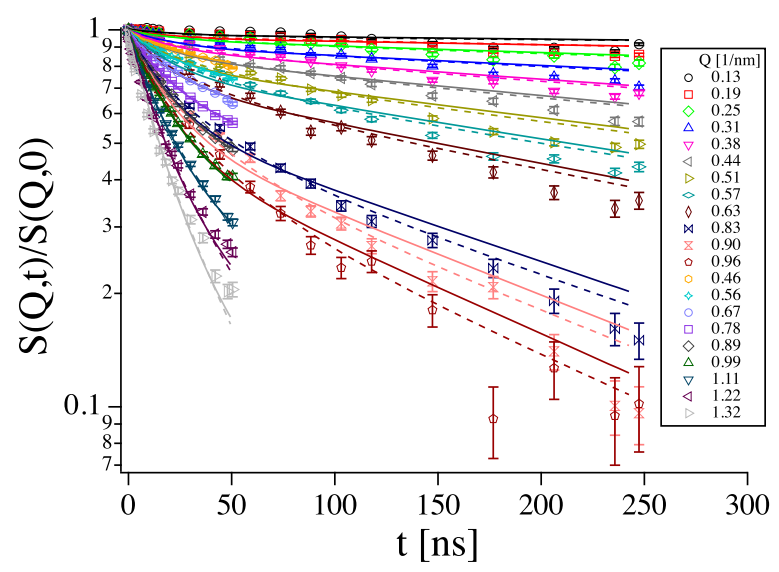

FIG. 5. $S(Q, t)$ of $80 \mathrm{mM}$ sodium oleate with 3 wt. $\% 225 \mathrm{mM}$ octanol; fits using the Milner-Safran model: Eq. (7) with Eq. (8), Eq. (10), and a simple exponential for $S_{\text {und }}$ (full lines) and terms up to $l=5$ according to Eq. (S3) and Eq. (11) (dashed lines); fits are in significantly worse agreement with the data than fits using the Zilman-Granek model, and the additional constant relaxation rate fails to describe the data over the whole $Q$ range.

The values obtained for $\kappa$ with the Zilman-Granek model, using an effective solvent viscosity of $\eta_{\text {eff }}=3 \eta$ to account for additional dissipation in the membrane, ${ }^{57-59}$ are shown in Fig. 7. It can be seen that $\kappa$ increases with $d_{\text {shell }}$. The obtained values for $\kappa$ are quite small and similar to values observed in microemulsions. ${ }^{49,61,66-72}$ However, it should be noted that the bilayers investigated here contain as majority component octanol and are with 2.0-2.2 nm thickness thinner by about a factor 2 compared with phospholipid bilayers. Accordingly, one would expect about a factor 4 to 8 lower values for $\kappa$. Another remarkable observation is the fact that (within the admittedly small range of $\left.d_{\text {shell }}\right) \kappa$ scales strongly with the shell thickness and both a scaling of $\kappa \propto d_{\text {shell }}^{2}$ and $\kappa \propto d_{\text {shell }}^{3}$ (dash-dotted and dashed line in Fig. 7) is too weak and the data rather support $\kappa \propto d_{\text {shell }}^{8}$ (full line). An explanation here would be that by adding octanol one does not only make the membrane thinner, but the increased presence of the short chain alcohol octanol also leads to an additional substantial

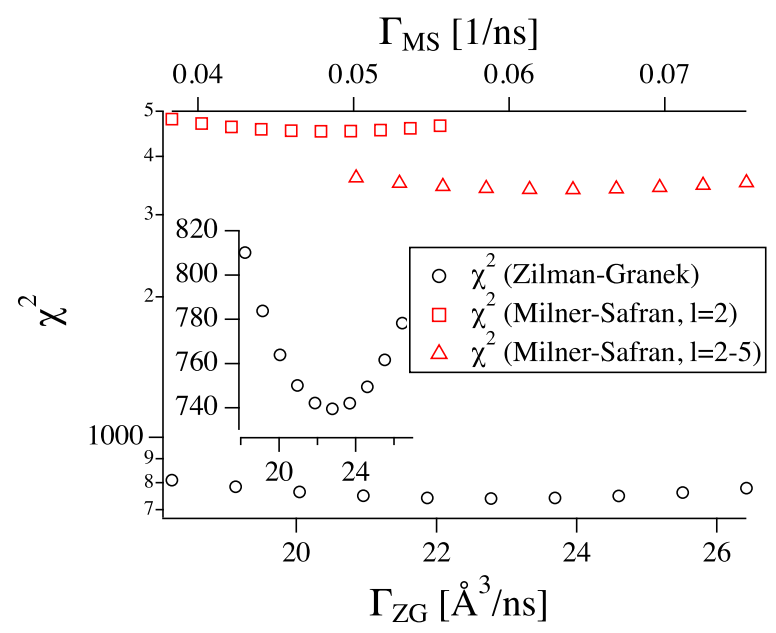

FIG. 6. $\chi^{2}$ obtained from fitting the Zilman-Granek and Milner-Safran (using only the $l=2$ term and terms up to $l=5, \Gamma_{M S}$ corresponds to the value for $l=2$ ) model to data from $80 \mathrm{mM}$ sodium oleate with $225 \mathrm{mM}$ octanol by varying the respective relaxation rate $\left(\Gamma_{Z G}\right.$ or $\left.\Gamma_{M S}\right)$ by $20 \%$ around their optima and optimising $A(Q)$; inset: $\chi^{2}$ for the Zilman-Granek model with linear axis. 


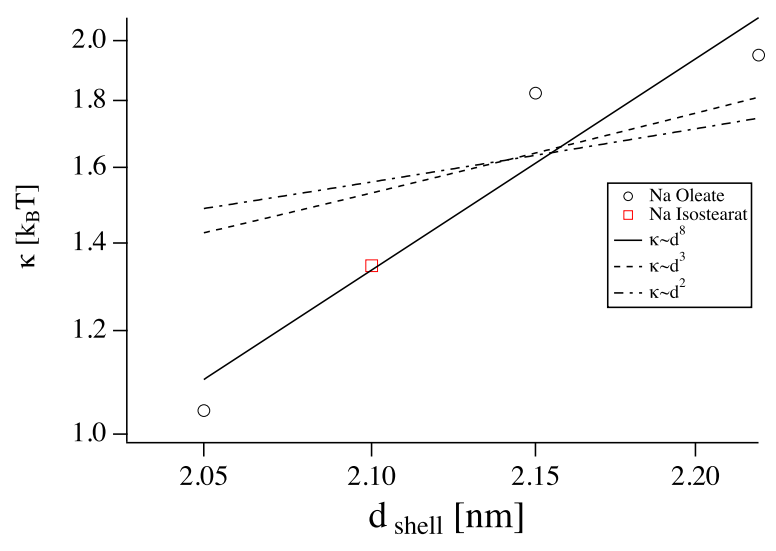

FIG. 7. Bending rigidity $\kappa$ of the bilayer as obtained from NSE measurements as a function of bilayer thickness $d_{\text {shell }}$. The bilayer is quite soft, similar to microemulsion systems, and $\kappa$ increases strongly with $d_{\text {shell }}$.

softening of the membrane. It might be noted here that, of course, the changes in the relaxation are not entirely due to the changing bending elasticity of the bilayers but also reflect changes of the internal dissipation (internal friction) in the membrane, as the ratio between surfactant and octanol changes here quite largely. However, we follow here the conventional notion and attribute the changes in stiffness to a changing bending rigidity, and our findings are also in principal agreement with theoretical calculations that indicate a softening of bilayers due to having a mixed bilayer of chains with largely different chain length. It might be argued that hydrodynamics [which are ignored in our analysis by using Eq. (8) and not $D(Q)=D_{0} \frac{H(Q)}{S(Q)}$ with the hydrodynamic function $\left.H(Q)\right]$ influence the obtained results, as at higher concentrations, the short time diffusion coefficient should be increasingly reduced as the high $Q$ limit of $H(Q)<1 .^{73-75}$ This would mean that the diffusional component calculated based on Eqs. (8) and (9) with the radius obtained from SANS would yield too high values of $D$ which would lead to too low values of $\Gamma_{Z G}$ (too high values of $\kappa$ ) with the effect being more pronounced for higher concentrations. However, it is found experimentally that $k$ decreases with concentration $\left(d_{\text {shell }}\right.$ decreases with concentration) and thus the change of $\kappa$ would be even more pronounced if we were accounting for hydrodynamic interactions in our analysis.

\section{CONCLUSION}

We have investigated the membrane dynamics of small unilamellar vesicles using neutron spin-echo (NSE) spectroscopy. While the Milner-Safran model is well suited for the description of the undulation motions of small droplet microemulsions, the Zilman-Granek model can be applied to the description of undulation motions in larger self assembled structures, such as large vesicles or bilayer structures or nanoemulsions. We found that even the relatively small unilamellar vesicles with radii between 20 and $40 \mathrm{~nm}$ used in this study are much better described by the Zilman-Granek model, while the Milner-Safran model leads to an unrealistic interpretation of the elastic properties of the bilayers. The bending rigidities found here for these small vesicles are in a similar range as those found for microemulsion droplets.
Therefore, for such low bending rigidities, the size below which the Milner-Safran model is better suited must be somewhere between 5 and $20 \mathrm{~nm}$.

It was also found that the bending rigidity $\kappa$ increases strongly with the thickness of the surfactant bilayer. While the layer thickness $d_{\text {shell }}$ changes by only about $10 \%$, the bending rigidity changes by a factor 2 and consequently the relaxation rate changes by a factor of $1 / \sqrt{2}$ as $\Gamma_{Z G} \propto 1 / \sqrt{\kappa}$. This very pronounced reduction of $\kappa$ upon addition of octanol cannot just be explained by the thinning of the membrane but must be associated with a marked softening due to the presence of this shorter chain molecules, which is in good qualitative agreement with calculations $\mathrm{s}^{35,76}$ of the bending rigidity based on chain entropy.

\section{SUPPLEMENTARY MATERIAL}

See supplementary material for additional formulas concerning the hard sphere structure factor and the Milner-Safran model and additional figures.

\section{ACKNOWLEDGMENTS}

Financial support from the BMBF Project No. 05K13KT1 is gratefully acknowledged as well as allocation of beamtime by ILL and HZB.

${ }^{1}$ M. Gradzielski, M. Müller, M. Bergmeier, H. Hoffmann, and E. Hoinkis, "Structural and macroscopic characterization of a gel phase of densely packed monodisperse, unilamellar vesicles," J. Phys. Chem. B 103, 14161424 (1999).

${ }^{2}$ G. Cevc, "Lipid vesicles and other colloids as drug carriers on the skin," Adv. Drug Delivery Rev. 56, 675-711 (2004).

${ }^{3}$ K. Morigaki and P. Walde, "Fatty acid vesicles," Curr. Opin. Colloid Interface Sci. 12, 75-80 (2007).

${ }^{4}$ G. El Maghraby, B. Barry, and A. Williams, "Liposomes and skin: From drug delivery to model membranes," Eur. J. Pharm. Sci. 34, 203-222 (2008).

${ }^{5}$ R. Rajera, K. Nagpal, S. K. Singh, and D. N. Mishra, "Niosomes: A controlled and novel drug delivery system,” Biol. Pharm. Bull. 34, 945-953 (2011).

${ }^{6}$ M. C. Moran, M. G. Miguel, and B. Lindman, "DNA gel particles from single and double-tail surfactants: Supramolecular assemblies and release characteristics," Soft Matter 7, 2001-2010 (2011).

${ }^{7}$ T. M. Allen and P. R. Cullis, "Liposomal drug delivery systems: From concept to clinical applications," Adv. Drug Delivery Rev. 65, 36-48 (2013).

${ }^{8}$ M. G. Basavaraj, N. L. McFarlane, M. L. Lynch, and N. J. Wagner, "Nanovesicle formation and microstructure in aqueous ditallowethylesterdimethylammonium chloride (DEEDMAC) solutions," J. Colloid Interface Sci. 429, 17-24 (2014).

${ }^{9}$ F. Liebig, A. F. Thünemann, and J. Koetz, "Ostwald ripening growth mechanism of gold nanotriangles in vesicular template phases," Langmuir 32, 10928-10935 (2016).

${ }^{10}$ D. D. Lasic, Liposomes: From Physics to Applications (Elsevier, Amsterdam, 1993).

${ }^{11}$ D. D. Lasic, "Novel applications of liposomes," Trends Biotechnol. 16, 307-321 (1998).

${ }^{12}$ B. Angelov, A. Angelova, M. Drechsler, V. M. Garamus, R. Mutafchieva, and S. Lesieur, "Identification of large channels in cationic pegylated cubosome nanoparticles by synchrotron radiation SAXS and Cryo-TEM imaging," Soft Matter 11, 3686-3692 (2015).

${ }^{13}$ W. Helfrich, "Effect of thermal undulations on the rigidity of fluid membranes and interfaces," J. Phys. 46, 1263-1268 (1985).

${ }^{14}$ W. Helfrich, "Elastic properties of lipid bilayers-Theory and possible experiments," Z. Naturforsch. C: J. Biosci. 28, 693-703 (1973).

${ }^{15}$ E. Evans and W. Rawicz, "Entropy-driven tension and bending elasticity in condensed-fluid membranes,” Phys. Rev. Lett. 64, 2094-2097 (1990). 
${ }^{16} \mathrm{H}$. Strey and M. Peterson, "Measurement of erythrocyte-membrane elasticity by flicker eigenmode decomposition," Biophys. J. 69, 478-488 (1995).

${ }^{17}$ E. Freyssingeas, D. Roux, and F. Nallet, "Quasi-elastic light scattering study of highly swollen lamellar and sponge phases," J. Phys. II 7, 913-929 (1997).

${ }^{18}$ M. Gradzielski, "Bending constants of surfactant layers," Curr. Opin. Colloid Interface Sci. 3, 478-484 (1998).

${ }^{19}$ P. Bassereau, B. Sorre, and A. Levy, "Bending lipid membranes: Experiments after W. Helfrich's model," Adv. Colloid Interface Sci. 208, 47-57 (2014).

${ }^{20}$ R. Dimova, "Recent developments in the field of bending rigidity measurements on membranes," Adv. Colloid Interface Sci. 208, 225-234 (2014).

${ }^{21}$ J. J. Pan, S. Tristram-Nagle, and J. F. Nagle, "Effect of cholesterol on structural and mechanical properties of membranes depends on lipid chain saturation," Phys. Rev. E 80, 021931 (2009).

${ }^{22}$ F. Mezei, "Neutron spin echo: A new concept in polarized thermal neutron techniques," Z. Phys. A: Hadrons Nucl. 255, 146-160 (1972).

${ }^{23}$ F. Mezei, in Neutron Spin Echo, Lecture Notes in Physics, edited by F. Mezei (Springer Berlin Heidelberg, 1980), Vol. 128, pp. 1-26.

${ }^{24}$ W. Pfeiffer, S. König, J. F. Legrand, T. Bayerl, D. Richter, and E. Sackmann, "Neutron spin echo study of membrane undulations in lipid multibilayers," Europhys. Lett. 23, 457 (1993)

${ }^{25}$ I. Hoffmann, "Neutrons for the study of dynamics in soft matter systems," Colloid Polym. Sci. 292, 2053-2069 (2014).

${ }^{26}$ W. Helfrich, "Lyotropic lamellar phases," J. Phys.: Condens. Matter 6, A79_ A92 (1994).

${ }^{27}$ M. Nagao, S. Chawang, and T. Hawa, "Interlayer distance dependence of thickness fluctuations in a swollen lamellar phase," Soft Matter 7, 65986605 (2011)

${ }^{28}$ M. Nagao, "Observation of local thickness fluctuations in surfactant membranes using neutron spin echo," Phys. Rev. E 80, 031606 (2009).

${ }^{29}$ B. S. Yang, J. Lal, M. Mihailescu, M. Monkenbusch, D. Richter, J. S. Huang, J. Kohn, W. B. Russel, and R. K. Prud'homme, "Neutron spin-echo study of dynamics of hydrophobically modified polymer-doped surfactant bilayers," Langmuir 18, 6-13 (2002).

${ }^{30}$ X. S. Hu, R. Biehl, R. K. Prud'homme, M. Monkenbusch, and J. Lal, "Fluctuations of bare membranes and their modification on incorporation of polymers having equally spaced anchors," Phys. B 350, 217-219 (2004).

${ }^{31}$ E. Freyssingeas, D. Roux, and F. Nallet, "The effect of water thickness on the bending rigidity of inverted bilayers," J. Phys.: Condens. Matter 8, 2801 (1996).

${ }^{32}$ H. Bermúdez, D. A. Hammer, and D. E. Discher, "Effect of bilayer thickness on membrane bending rigidity," Langmuir 20, 540-543 (2003).

${ }^{33}$ L. D. Landau and E. M. Lifshitz, Theory of Elasticity, 2nd English ed., Volume 7 of Course of Theoretical Physics (Pergamon Press, 1970).

${ }^{34}$ E. A. Evans, "Bending resistance and chemically-induced moments in membrane bilayers," Biophys. J. 14, 923-931 (1974).

${ }^{35}$ I. Szleifer, D. Kramer, A. Ben-Shaul, D. Roux, and W. M. Gelbart, "Curvature elasticity of pure and mixed surfactant films," Phys. Rev. Lett. 60, 1966-1969 (1988).

${ }^{36}$ M. Mell, L. Moleiro, Y. Hertle, P. Fouquet, R. Schweins, I. López-Montero, T. Hellweg, and F. Monroy, "Bending stiffness of biological membranes: What can be measured by neutron spin echo?," Eur. Phys. J. E 36, 75 (2013).

${ }^{37}$ S. A. Safran, "Fluctuations of spherical microemulsions," J. Chem. Phys. 78, 2073-2076 (1983)

${ }^{38}$ S. T. Milner and S. A. Safran, "Dynamical fluctuations of droplet microemulsions and vesicles," Phys. Rev. A 36, 4371-4379 (1987).

${ }^{39}$ M. Gradzielski, M. Bergmeier, M. Muller, and H. Hoffmann, "Novel gel phase: A cubic phase of densely packed monodisperse, unilamellar vesicles,” J. Phys. Chem. B 101, 1719-1722 (1997).

${ }^{40}$ M. Gradzielski, M. Bergmeier, H. Hoffmann, M. Muller, and I. Grillo, "Vesicle gel formed by a self-organization process," J. Phys. Chem. B 104, 11594-11597 (2000).

${ }^{41}$ K. Fontell, L. Mandell, and P. Ekwall, "Some isotropic mesophases in systems containing amphiphilic compounds," Acta Chem. Scand. 22, 3209 (1968).

${ }^{42}$ Neutrons, $X$-rays and Light: Scattering Methods Applied to Soft Condensed Matter, edited by P. Lindner and T. Zemb (Elsevier Science Ltd., 2002).

${ }^{43} \mathrm{U}$. Keiderling and A. Wiedenmann, "New SANS instrument at the BER-II reactor in Berlin, Germany," Phys. B: Condens. Matter 213-214, 895-897 (1995).
${ }^{44}$ R. Gilles, U. Keiderling, P. Strunz, A. Wiedenmann, and H. Fuess, "Silver behenate as a standard for instrumental resolution and wavelength calibration for small angle neutron scattering," Mater. Sci. Forum 321-324, 264-269 (2000).

${ }^{45} \mathrm{U}$. Keiderling, "The new 'BerSANS-PC' software for reduction and treatment of small angle neutron scattering data," Appl. Phys. A: Mater. Sci. Process. 74, S1455-S1457 (2002).

${ }^{46}$ J. S. Pedersen, "Determination of size distribution from small-angle scattering data for systems with effective hard-sphere interactions," J. Appl. Crystallogr. 27, 595-608 (1994).

${ }^{47}$ J. K. Percus and G. J. Yevick, "Analysis of classical statistical mechanics by means of collective coordinates," Phys. Rev. 110, 1-13 (1958).

${ }^{48}$ P. Schleger, B. Alefeld, J. Barthelemy, G. Ehlers, B. Farago, P. Giraud, C. Hayes, A. Kollmar, C. Lartigue, F. Mezei, and D. Richter, "The longwavelength neutron spin-echo spectrometer IN15 at the Institut LaueLangevin," Phys. B 241-243, 164-165 (1997).

${ }^{49}$ B. Farago, D. Richter, J. S. Huang, S. A. Safran, and S. T. Milner, "Shape and size fluctuations of microemulsion droplets: The role of cosurfactant," Phys. Rev. Lett. 65, 3348-3351 (1990).

${ }^{50} \mathrm{M}$. Gradzielski, D. Langevin, and B. Farago, "Experimental investigation of the structure of nonionic microemulsions and their relation to the bending elasticity of the amphiphilic film," Phys. Rev. E 53, 3900-3919 (1996).

${ }^{51}$ T. Hellweg and D. Langevin, "Bending elasticity of the surfactant monolayer in droplet microemulsions: Determination by a combination of dynamic light scattering and neutron spin-echo spectroscopy," Phys. Rev. E 57, 68256834 (1998).

${ }^{52}$ B. Farago and M. Gradzielski, "The effect of the charge density of microemulsion droplets on the bending elasticity of their amphiphilic film," J. Chem. Phys. 114, 10105-10122 (2001).

${ }^{53}$ L. R. Arriaga, I. López-Montero, F. Monroy, G. Orts-Gil, B. Farago, and T. Hellweg, "Stiffening effect of cholesterol on disordered lipid phases: A combined neutron spin echo + dynamic light scattering analysis of the bending elasticity of large unilamellar vesicles," Biophys. J. 96, 3629-3637 (2009).

${ }^{54}$ L. R. Arriaga, I. López-Montero, G. Orts-Gil, B. Farago, T. Hellweg, and F. Monroy, "Fluctuation dynamics of spherical vesicles: Frustration of regular bulk dissipation into subdiffusive relaxation," Phys. Rev. E 80, 031908 (2009).

55 A. G. Zilman and R. Granek, "Undulations and dynamic structure factor of membranes," Phys. Rev. Lett. 77, 4788-4791 (1996).

${ }^{56}$ A. G. Zilman and R. Granek, "Membrane dynamics and structure factor," Chem. Phys. 284, 195-204 (2002).

${ }^{57}$ B. Farago, M. Monkenbusch, K. Goecking, D. Richter, and J. Huang, "Dynamics of microemulsions as seen by neutron spin echo," Phys. B 213-214, 712-717 (1995).

${ }^{58}$ S. Komura, T. Takeda, Y. Kawabata, S. K. Ghosh, H. Seto, and M. Nagao, "Dynamical fluctuation of the mesoscopic structure in ternary $c_{12} e_{5}$-watern-octane amphiphilic systems," Phys. Rev. E 63, 041402 (2001).

${ }^{59}$ I. Hoffmann, P. Heunemann, B. Farago, I. Grillo, O. Holderer, M. Päch, and M. Gradzielski, "Structure and dynamics of nanoemulsions: Insights from combining dynamic and static neutron scattering," Phys. Rev. E 86, 061407 (2012)

${ }^{60}$ U. Seifert and S. A. Langer, "Viscous modes of fluid bilayer membranes," Europhys. Lett. 23, 71-76 (1993).

${ }^{61}$ M. Monkenbusch, O. Holderer, H. Frielinghaus, D. Byelov, J. Allgaier, and D. Richter, "Bending moduli of microemulsions; comparison of results from small angle neutron scattering and neutron spin-echo spectroscopy," J. Phys.: Condens. Matter 17, S2903-S2909 (2005).

${ }^{62}$ M. C. Watson and F. L. H. Brown, "Interpreting membrane scattering experiments at the mesoscale: The contribution of dissipation within the bilayer," Biophys. J. 98, L9-L11 (2010).

${ }^{63}$ M. C. Watson, Y. Peng, Y. Zheng, and F. L. H. Brown, "The intermediate scattering function for lipid bilayer membranes: From nanometers to microns," J. Chem. Phys. 135, 194701 (2011).

${ }^{64}$ L. R. Arriaga, R. Rodríguez-García, L. H. Moleiro, S. Prévost, I. LópezMontero, T. Hellweg, and F. Monroy, "Dissipative dynamics of fluid lipid membranes enriched in cholesterol," Adv. Colloid Interface Sci. 247, 514 520 (2017).

${ }^{65}$ P. G. De Gennes, "Liquid dynamics and inelastic scattering of neutrons," Physica 25, 825-839 (1959).

${ }^{66} \mathrm{~T}$. Sottmann, "Solubilization efficiency boosting by amphiphilic block copolymers in microemulsions," Curr. Opin. Colloid Interface Sci. 7, 57-65 (2002) 
${ }^{67}$ M. Mihailescu, M. Monkenbusch, J. Allgaier, H. Frielinghaus, D. Richter, B. Jakobs, and T. Sottmann, "Neutron scattering study on the structure and dynamics of oriented lamellar phase microemulsions," Phys. Rev. E 66, 041504 (2002).

${ }^{68} \mathrm{M}$. Nagao and H. Seto, "Concentration dependence of shape and structure fluctuations of droplet microemulsions investigated by neutron spin echo spectroscopy," Phys. Rev. E 78, 011507 (2008).

${ }^{69}$ T. Foster, T. Sottmann, R. Schweins, and R. Strey, "Small-angle-neutronscattering from giant water-in-oil microemulsion droplets. II. Polymerdecorated droplets in a quaternary system," J. Chem. Phys. 128, 064902 (2008).

${ }^{70}$ S. Wellert, M. Karg, O. Holderer, A. Richardt, and T. Hellweg, "Temperature dependence of the surfactant film bending elasticity in a bicontinuous sugar surfactant based microemulsion: A quasielastic scattering study," Phys. Chem. Chem. Phys. 13, 3092-3099 (2011).

${ }^{71}$ I. Hoffmann, P. Malo De Molina, B. Farago, P. Falus, C. Herfurth, A. Laschewsky, and M. Gradzielski, "Dynamics of microemulsions bridged with hydrophobically end-capped star polymers studied by neutron spinecho," J. Chem. Phys. 140, 034902 (2014).

${ }^{72}$ B. Kuttich, P. Falus, I. Grillo, and B. Stühn, "Form fluctuations of polymer loaded spherical microemulsions studied by neutron scattering and dielectric spectroscopy," J. Chem. Phys. 141, 084903 (2014).

${ }^{73}$ C. W. J. Beenakker and P. Mazur, "Diffusion of spheres in a concentrated suspension-Resummation of many-body hydrodynamic interactions," Phys. Lett. A 98, 22-24 (1983).

${ }^{74}$ L. Porcar, P. Falus, W. Chen, A. Faraone, E. Fratini, K. Hong, P. Baglioni, and Y. Liu, "Formation of the dynamic clusters in concentrated lysozyme protein solutions," J. Phys. Chem. Lett. 1, 126-129 (2010).

${ }^{75}$ P. Falus, L. Porcar, E. Fratini, W. Chen, A. Faraone, K. Hong, P. Baglioni, and Y. Liu, "Distinguishing the monomer to cluster phase transition in concentrated lysozyme solutions by studying the temperature dependence of the short-time dynamics," J. Phys.: Condens. Matter 24, 064114 (2012).

${ }^{76}$ I. Szleifer, D. Kramer, A. Ben-Shaul, W. M. Gelbart, and S. A. Safran, "Molecular theory of curvature elasticity in surfactant films," J. Chem. Phys. 92, 6800-6817 (1990). 